Канте Н.Б., Лавендел Ю.О.

\title{
ПОЛУЧЕНИЕ ПЛАНА ЭКСПЕРИМЕНТОВ, НА ОСНОВЕ МОДЕЛИРОВАНИЯ ВЗАИМОДЕЙСТВИЯ ЗАРЯЖЕННЫХ ЧАСТИЦ
}

\author{
Рижский Технический Университет
}

\begin{abstract}
Аннотация
Рассмотрен метод по получению плана экспериментов в многомерном пространстве. Метод основан на предположениях о равномерном распределении заряженных частии в бесконечном пространстве. Чтобы получить план экспериментов, бесконечное многомерное пространство заменяется гиперкубом, поверхность которого моделирует воздействие бесконечного пространства. Разработано программное обеспечение и получены практические результаты, в двумерном пространстве. Нет принципиальных проблем провести расчёты и в многомерном пространстве.
\end{abstract}

Ключевые слова: План экспериментов, взаимодействия заряженных частиц, Латинский квадрат.

Kant N.B., Lavendel Yu.O.

\section{OBTAINING THE PLAN OF EXPERIMENTS, ON THE BASIS OF MODELLING OF INTERACTION OF CHARGED PARTICLES}

\author{
Riga Technical University
}

\begin{abstract}
The method on obtaining the plan of experiments in multidimensional space is considered. The method is based on assumptions of uniform distribution of charged particles in infinite space. To receive the plan of experiments, the infinite multidimensional space is replaced with a hyper cube which surface models influence of infinite space. The software is developed and practical results, in two-dimensional space are received. There are no basic problems to carry out calculations and in multidimensional space.
\end{abstract}

Keywords: Plan of experiments, interactions of charged particles, Latin square.

\section{Введение}

В работе технических, в частности тренажёрных системах, реальный технический или природный процесс заменяется математической моделью. Модель часто представляет набор характеристик процесса в отдельных точках расчётной области точках плана эксперимента [1]. Значения характеристик процесса являются результатами расчёта, например, численным решением уравнения математической физики или результатом метамоделирования. Характеристики процесса вне точек плана эксперимента находятся методами интерполирования, регрессии или другими приближёнными расчётами. Точность замены процесса моделью процесса во многом определяется планом эксперимента.

Данная статья рассматривает один возможный подход к построению плана эксперимента.

Получение плана экспериментов, как правило, коренится на основе метода Латинского квадрата [2, 3]. Как правило, латинский квадрат в опытно-конструкторских исследованиях используется для получения плана экспериментов ив случае от 2 до 4 
независимых параметров. В современных тренажёрных технологиях требуется получение плана экспериментов с 8 - 10 или более независимых переменных (10-мерном пространстве), где метод латинских квадратов теряет свою эффективность. Следовательно, актуальными становится другие подходы для получения плана экспериментов. В 1977 году ученный Вильнис Еглаис РПИ (Рижский Политехнический Институт) выдвинул гипотезу о том, что план экспериментов можно построить на основе анализа координат заряженных частиц в устойчивом состоянии [4].

В данной статье рассматривается один из возможных способов получения плана экспериментов, моделируя размещения заряженных частиц в экспериментальном пространстве.

\section{Схема расчёта}

В бесконечно вязком, н-мерном пространстве с определенной плотностью заряженных частиц, которые взаимодействуя друг с другом и периоде длительного времени достигается устойчивое состояние. Все движения прекращаются - система достигает состояния покоя. Из такого пространства вырезанный гиперкуб и был бы хорошим планом экспериментов.

Такого рода решение невозможно реализовать, не в физической, не в математической модели. В реалии же, заряженные частицы возможно исследовать в гиперкубе, в котором находятся заряженные частицы, и поверхность куба заменяет воздействие бесконечного пространства на гиперкуб. На рисунке 1 показана схема исследования двумерного (квадрат) случая.

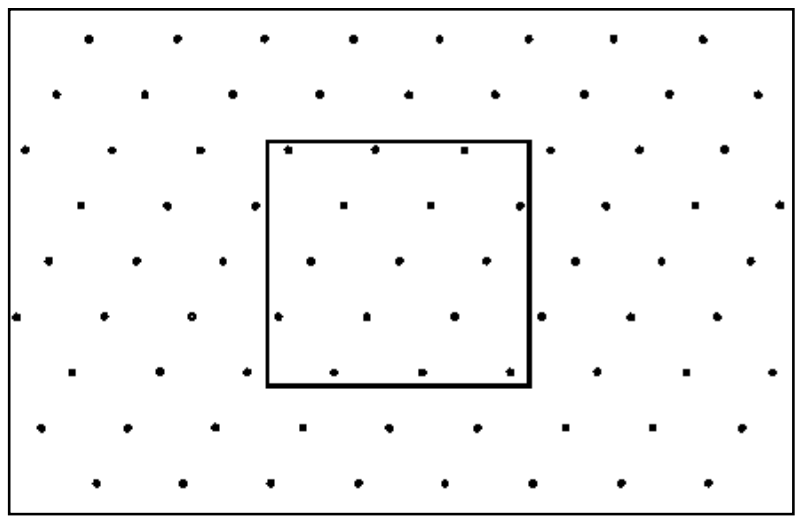

a.

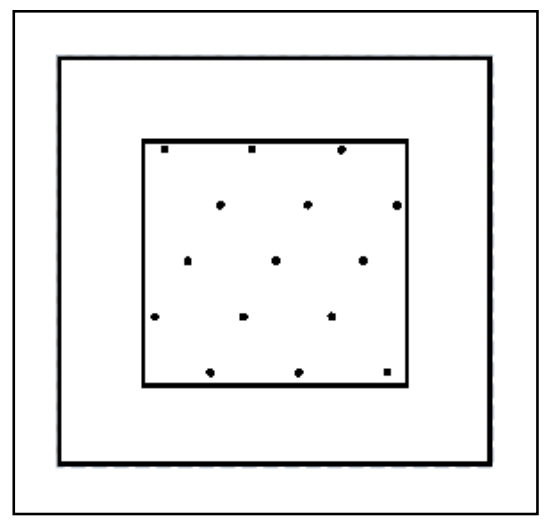

6.

Рисунок 1 - Схема расчёта

a. Фрагмент бесконечного двухмерного пространства с заряженными частицами, которые могут быть использованы в плане эксперимента.

б. Квадрат, и зона вокруг квадрата, которая «заменяет» воздействие бесконечного пространства.

Рассматривается квадрат, в котором находится определённое число заряженных частиц. Вокруг этого квадрата размещаются такие же квадраты с аналогичным распределением частиц - фантомов. Можно размещать 1, 2 или больше фантомных полос вокруг расчётного пространства. Отметим, что от числа полос зависит качество полученных результатов (рисунок 2). 


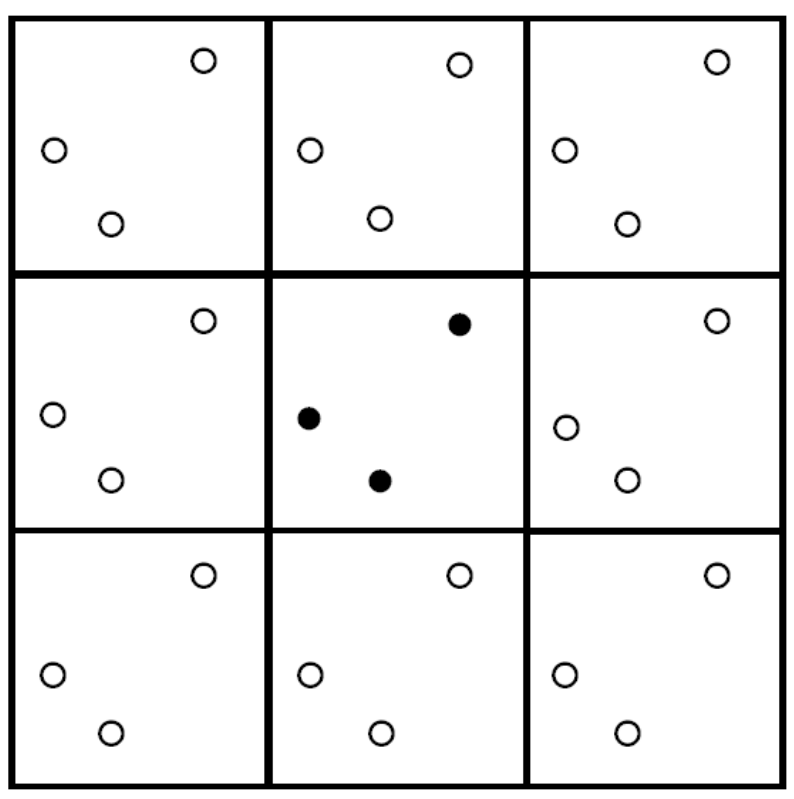

Рисунок 2 - Расчётная схема с одной фантомной полосой - - заряженные частицы о - фантомы заряженных частиц

Начально все заряженные частицы, расположенные в произвольном положении и имеют координаты, например, начальное состояние может быть получено генератором случайных чисел, сгенерировав координаты частиц в расчётной площади. Координаты фантомных частиц, рассчитываются исходя из выбранной модели расчётной области.

Всем частицам в расчётной области выполняются следующие действия: 3. a);

1. Определяется частица, для которой необходимо провести расчёт (Рисунок

2. Принимая во внимание при расчёте воздействие всех заряженных частиц расчётной области и всех частиц фантомов, рассчитываются новые координаты выделенной частицы, где суммарная сила на частицу равняется нулю (Рисунок 3. б);

3. В соответствии с новыми координатами частицы, регулируются координаты соответствующих фантомных частиц (Рисунок 3. в);

4. Возвращение к пункту 1.

Определение координат частицы это повторяющийся процесс, которий продолжается до тех пор, пока сила взаимодействующих частиц системы минимизируется. Необходимо отметить, что не любое количество частиц в области, даёт возможность получить визуально красивый результат. Но практическое применение наших расчётов, применимо.

Все расчёты для каждой частицы производятся по принципу суперпозиции по каждой координате отдельно. 


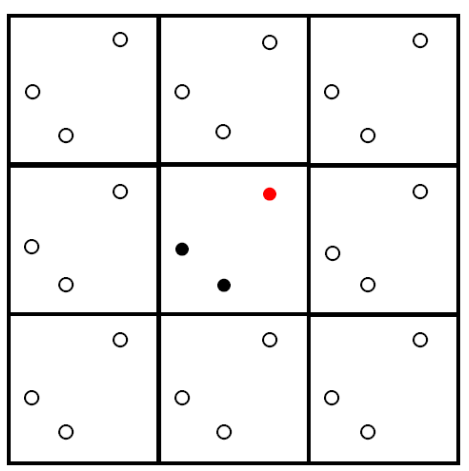

a.

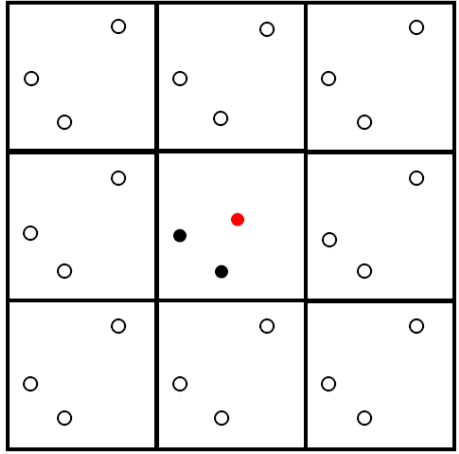

б.

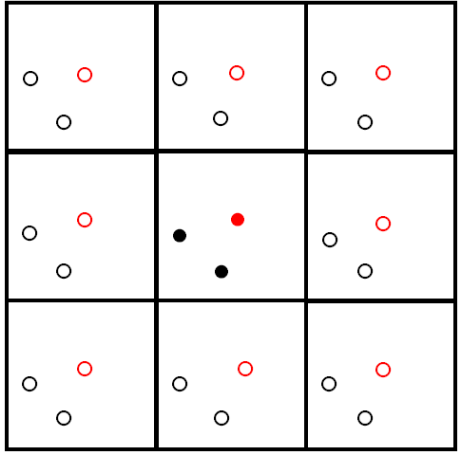

B.

Рисунок 3 - Моделирование размещение частиц

a - Исходное положение, и выбор «перемещаемой» частицы;

б - Определение новых координат перемещаемой частицы;

в - коррекция координат фантомных частиц.

\section{Практические результаты}

Рассмотренная методология была реализована на практике, при помощи разработанного программного обеспечения. Были проведены практические вычисления для получения плана экспериментов, которые представлены на рисунке 4.

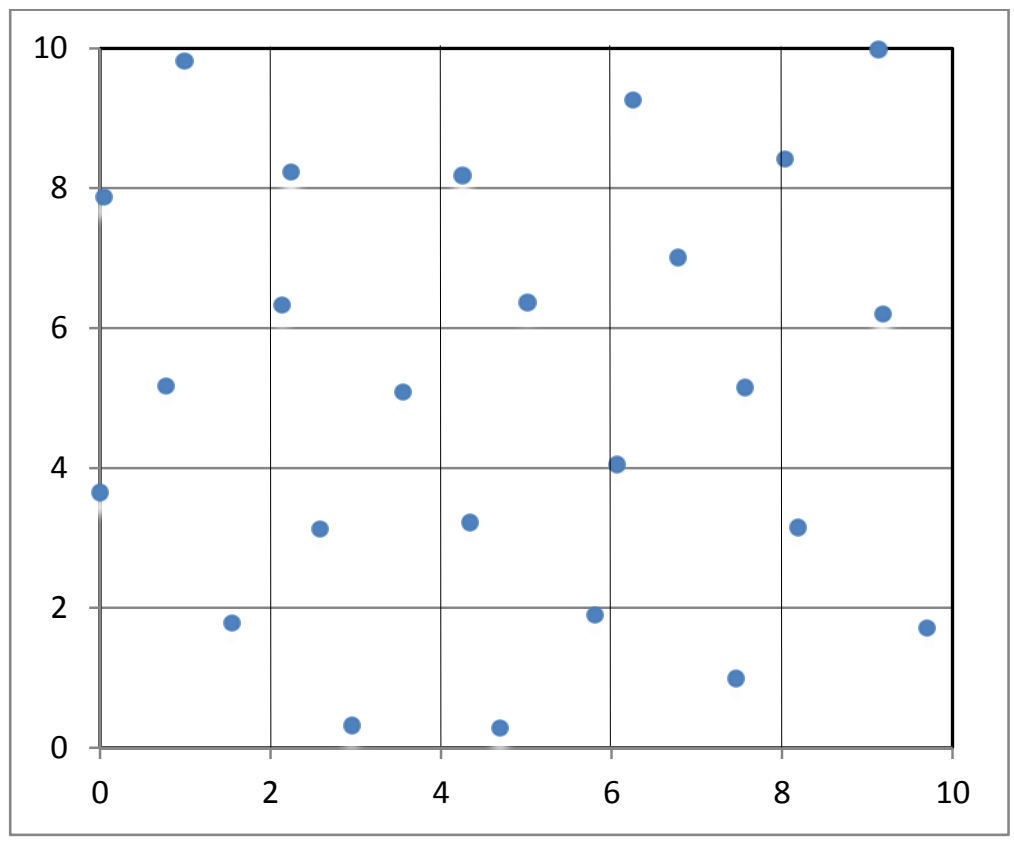

Рисунок 4 - Пример плана эксперимента - 25 экспериментальных точек в двухмерном квадрате

\section{Окончание}

Практически реализован метод получения плана экспериментов, который основан на моделировании поведения заряженных частиц. Разработанное программное обеспечение применимо на практике, наряду с другими методами. Разработанный метод был реализован в двухмерном случае. Его также легко реализовать с большим количеством измерений, поскольку используя суперпозицию, каждая координата рассчитывается отдельно.

Метод является применимым для плана экспериментов с неравномерным 
распределением частиц, что делает возможным, например, выделить в тренажёрных системах регионы с «большей» точностью. В этом случае заряд частиц задают как функцию координат.

Получены приемлемые результаты, которие подтверждают жизнеспособность подхода.

\section{Библиографический список}

1. Auzins J., Janusevskis А. «Планирование и анализ экспериментов», Рига, РТУ, (на латышском языке), 2007

2. Butler N.A. «Optimal and Orthogonal Latin Hypercube Designs for Computer Experiements». Biometrika 88, 2001, $847-857$

3. Iman R.J. and Shortencarier M.J., A FORTRAN77 «Program and User's Guide for Generation of Latin Hypercube and Random Samples for Use with Computer Models» NUREG/CR-3624, SAND83-2365, Sandia National Laboratories, Albuquerque, NM, 1984

4. Audze P. and Eglājs V. «New approach to the design of multifactor experiments» Problems of Dynamics and Strengths 35, Zinatne Publishing House, Riga, 1977, 104 - 107 